\title{
Outcome of depressive mood disorder among adolescent outpatients in a 8-year follow-up
}

\author{
Olli Kiviruusua,b, Thea Strandholma , Linnea Karlssonn,d, Mauri Marttunenª,b \\ a Psychiatry, University of Helsinki and Helsinki University Hospital, Helsinki, Finland \\ b Department of Public Health Solutions, National Institute for Health and Welfare, Helsinki, Finland \\ c FinnBrain Birth Cohort Study, Department of Clinical Medicine, Turku Brain and Mind Center, University of Turku, Turku, Finland \\ d Department of Child Psychiatry, University of Turku and Turku University Hospital, Turku, Finland
}

Objective. To investigate the 8-year course and outcome of depressive mood disorders and the key outcome predictors among adolescent outpatients across the important transition period into young adulthood.

Materials and methods. Data were drawn from the Adolescent Depression Study (ADS), a Finnish naturalistic clinical follow-up study. Depressive outpatients $(\mathrm{N}=148,13-$ $18 \mathrm{yrs}, 82 \%$ females) were assessed at baseline, 6 month, 12 month and 8 years using diagnostic and self-report instruments. Baseline predictors covered sociodemographic, clinical and treatment characteristics. Outcomes were time to recovery, recurrence, time spent ill and longitudinal latent depressive symptom profiles that were based on the Hamilton Depression Rating Scale (HDRS) scores assessed at each measurement point.

Results. Median length of index episode was 111 weeks (Q1=57, Q3=210 weeks) and time to recovery from baseline 57 weeks (Q1=28, Q3=113 weeks). Recovery rate from a depressive mood disorder was $73 \%$ at 2 years, $91 \%$ at 5 years and $94 \%$ by the end of mean 8 -year follow-up. One or more recurrent episodes were presented by $67 \%$ of the outpatients. Over half $(57 \%)$ of subjects spent $25 \%$ or more of the 8 -year follow-up period as depressed, median time spent ill being 126 weeks (Q1=71, Q3=251 weeks).
Comorbid diagnoses were common at baseline and at the 8-year follow-up (Table 1). At the 8-year follow-up, 36\% still had a mood disorder. Latent profile analyses based on HDRS scores suggested a three profile solution. The profile solution along with the significant baseline predictors from multivariate logistic regression analyses are depicted in Figure 1.

Conclusions. While for others the adolescent depression outcome into young adulthood years seems promising, for many episodes are long, even chronic, and recurrences are common. Comorbid psychiatric diagnoses were among the strongest predictors of the more severe and chronic depressive symptom profiles.

Table 1. Psychiatric comorbidity.

\begin{tabular}{|c|c|c|}
\hline Comorbid diagnose & Baseline & 8-year \\
\hline Anxiety disorder & $56 \%$ & $48 \%$ \\
\hline Substance use disorder & $13 \%$ & $10 \%$ \\
\hline Disruptive disorder & $10 \%$ & $1 \%$ \\
\hline Eating disorder & $10 \%$ & $7 \%$ \\
\hline \multicolumn{3}{|l|}{ Personality disorders } \\
\hline Borderline & $12 \%$ & $6 \%$ \\
\hline Other personality do & $33 \%$ & $21 \%$ \\
\hline
\end{tabular}

Figure 1. Latent profiles of HDRS scores at baseline, 6 and 12 months, and at 8-year follow-up.

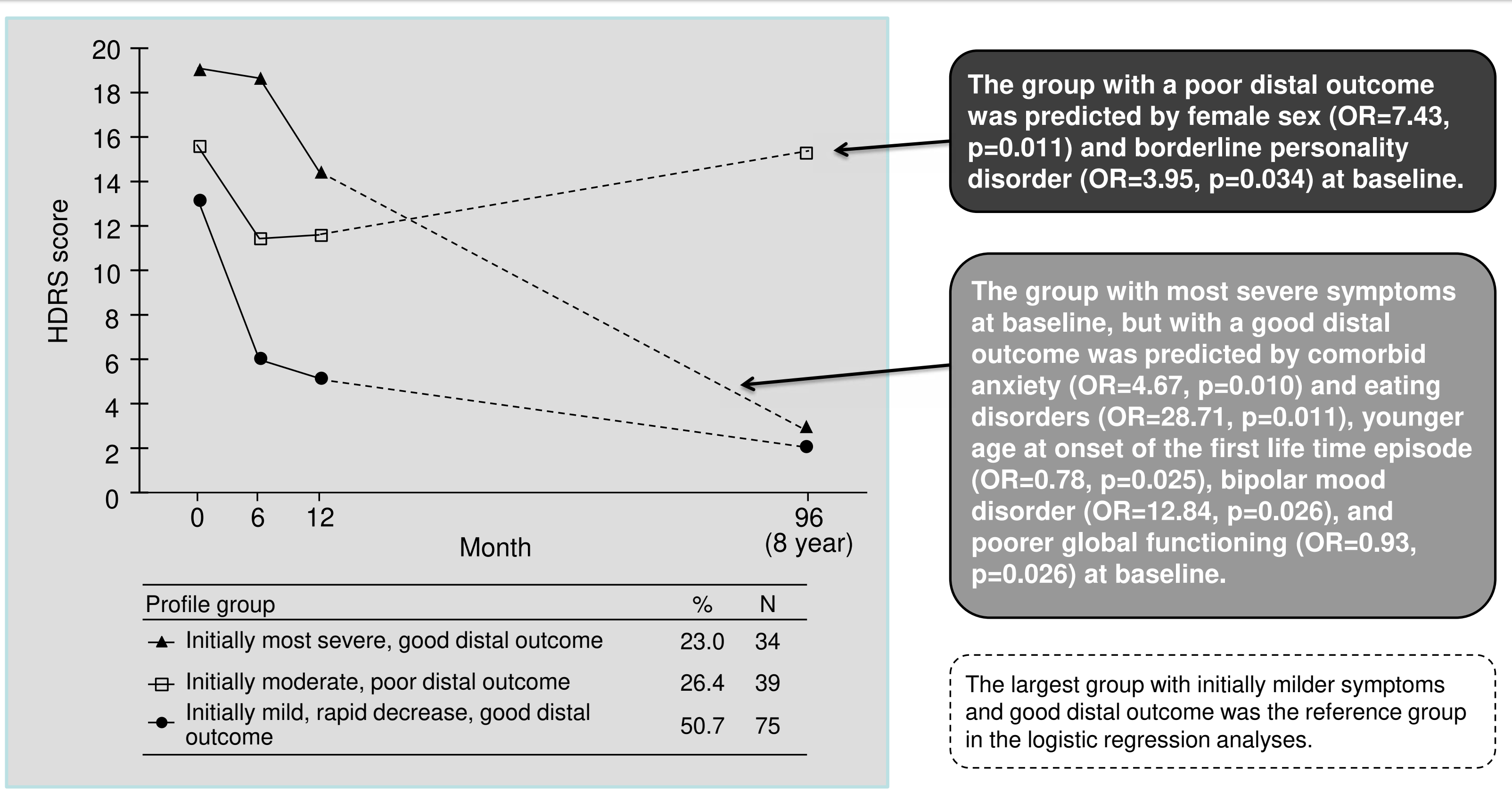

